\title{
CLEC-38, A Transmembrane Protein with C-Type Lectin- Like Domains, Negatively Regulates UNC-40-Mediated Axon Outgrowth and Promotes Presynaptic Development in Caenorhabditis elegans
}

\author{
Gauri Kulkarni, ${ }^{\star}$ Haichang Li, ${ }^{\star}$ and William G. Wadsworth \\ Department of Pathology and Laboratory Medicine, University of Medicine and Dentistry of New Jersey, Robert Wood Johnson Medical School, Piscataway, \\ New Jersey 08854-5636
}

\begin{abstract}
In the developing nervous system, axons respond to various guidance cues to find their targets. The effects guidance cues have on an axon may change as an axon undergoes morphological changes, such as branching, turning, and synapse formation. The means by which these changes are regulated are not well understood. In Caenorhabditis elegans, the UNC-40/DCC (deleted in colorectal cancer) receptor mediates responses to the UNC-6/netrin guidance cue. Here, we show that CLEC-38, a protein with predicted transmembrane and C-type lectin-like domains, regulates UNC-40-mediated axon outgrowth as well as the organization of presynaptic terminals. We observe that, in genetic backgrounds sensitized for axon guidance defects, loss of clec-38 function can suppress defects in an UNC-40-dependent manner. Within migrating axons, clec-38 acts cell autonomously. Furthermore, loss of clec-38 function alters UNC-40::GFP (green fluorescent protein) expression. We also observe that loss of $c l e c-38$ function disrupts presynaptic patterning in animals with normal axon guidance and that there are genetic interactions between $c l e c-38$ and $r p m-1$, which encodes a protein implicated in regulating presynaptic assembly and axon morphology. We suggest CLEC-38 plays a role in promoting synapse assembly and refining axon outgrowth activity.
\end{abstract}

Key words: axon guidance; C. elegans; development; genetics; growth cone; receptor; synaptogenesis

\section{Introduction}

In the developing nervous system migrating axons encounter multiple extracellular cues that help control the development of the axon. For example, axons that express the UNC-40/deleted in colorectal cancer (DCC), UNC-5/UNC5, and SAX-3/robo receptors are guided by extracellular UNC-6/netrin and SLT-1/slit cues toward their targets (Tessier-Lavigne and Goodman, 1996; Dickson, 2002). As different local environments are encountered, the response to these cues may change as axons are influenced by additional factors that stimulate new events such as branch formation and synapse development. These factors are mostly unknown, but may include the guidance cues and receptors themselves. For example, Slit and Robo can regulate axon guidance and stimulate branching in vertebrates (Wang et al., 1999; Ma and Tessier-Lavigne, 2007). In Caenorhabditis elegans, changing spatial and temporal patterns of UNC- 6 expression by neuroglia

Received Sept. 17, 2007; revised March 18, 2008; accepted March 22, 2008.

This work was supported by National Institutes of Health Grant R01 NS033156 and grants from the New Jersey Commission on Spinal Cord Research. We thank S. Clark, J. Culotti, C. Bargmann, Yishi Jin, the Japanese National BioResource Project, and the Caenorhabditis Genetics Center for strains; we also thank members of the Wadsworth Laboratory for constructive input, and Sunita Kramer, Christopher Quinn, and Martha Soto for critical discussion and comments on this manuscript.

${ }^{*}$ G.K. and H.L. contributed equally to this work.

Correspondence should be addressed to Dr. William G. Wadsworth, Department of Pathology, Robert Wood Johnson Medical School, 657 Hoes Lane West, Piscataway, NJ 08854-5635. E-mail: william.wadsworth@umdnj.edu. DOI:10.1523/JNEUROSCI.5542-07.2008

Copyright $\odot 2008$ Society for Neuroscience $\quad 0270-6474 / 08 / 284541-10 \$ 15.00 / 0$ and pioneer neurons indicate a hierarchy of netrin cues in the developing nervous system (Wadsworth et al., 1996). The phenotypes of unc-6 mutants, as well as laser ablation studies of UNC6-expressing cells, indicate that the UNC-6 cues direct local axon migrations as well as interactions among axons and glia cells during the assembly of the axon scaffold (Wadsworth and Hedgecock, 1996; Ren et al., 1999; Hutter, 2003). Within the developing nerve ring, it has been shown that local UNC-6/netrin cues and the UNC-40 receptor help guide axons as well as mediate the assembly of presynaptic terminals (Colon-Ramos et al., 2007).

We present here evidence that CLEC-38 can negatively regulate UNC-40-mediated outgrowth in axons migrating toward their targets. Furthermore, CLEC-38 is required for proper presynaptic development in axons that have reached their targets. To our knowledge, this is the first time that a member of the protein superfamily containing the C-type lectin-like domains (CTLDs) has been directly implicated in these processes. Based on our observations, we suggest that CLEC-38 acts in migrating axons to help mediate signals that both promote synaptogenesis and modify further UNC-40-mediated axon outgrowth.

\section{Materials and Methods}

Strains. Worms were cultivated according to standard protocol and were maintained at $20^{\circ} \mathrm{C}$ (Brenner, 1974).

N2, Bristol strain, was used as a standard wild-type strain. All mutations used for this study are strong loss-of-function or null alleles unless otherwise indicated. Strains constructed and used for this study are as 
follows: IM721: $\quad$ clec-38(ur280)V, IM720: clec-38(ur280) V; evIs82a[punc-129::GFP]IV, IM723: clec-38(tm2035)V, IM658: clec38(ur280)V; unc-6(rh46)X; evIs82aIV, IM660: clec-38(ur280)V; unc6(e78)X; evIs82a IV, IM661: unc-6(rh46)X; evIs82aIV, IM662: unc6(e78)X; evIs82aIV, IM207: unc-6(ev400)X; evIs82aIV, IM831: clec38(ur280)V; unc-6(ev400)X; evIs82aIV, IM832: unc-40(e1430)I; evIs82aIV, IM833: clec-38(ur280)V; unc40(e1430)I; evIs82aIV, IM834: unc-5(e53)IV evIs82aIV, IM835: clec-38(ur280)V; unc-5(e53)IV; evIs82aIV, IM869: clec-38(ur280)V; unc-40(e1430)I; unc-6(rh46)X; evIs82aIV, IM984: clec-38(ur280)V; unc-5(e53)IV; unc-6(rh46)X; evIs82aIV, IM682: clec-38(ur280)V; zdIs5[pmec-4::GFP]I, IM838: unc6(rh46)X; zdIs5I, IM836: clec-38(ur280)V; unc-6(rh46)X; zdIs5I, IM650: unc-6(ev400)X; zdIs5I, IM837: clec-38(ur280)V; unc-6(ev400)X; zdIs5I, IM647: slt-1(eh15); zdIs5, IM839: clec-38(ur280)V; slt1(eh15); zdIs5I, IM649: unc-6(ev400)X; slt-1(eh15)X; zdIs5I, IM712: sax-3(ky123)X; zdIs5I, IM713: clec-38(ur280)V; sax-3(ky123)X; zdIs5I, IM739: unc5(e53)IV; zdIs5I, IM648: unc-40(e1430)I; zdIs5I, IM840: clec-38(ur280)V; unc40(e1430)I; zdIs5I, IM843: rpm-1(ur299)V, IM844: rpm-1(ur299)V; evIs82aIV, IM805: rpm-1(ur299)V; unc-6(rh46)X, IM893: clec38(ur280)V; rpm-1(ur299)V; unc-6(rh46)X; evIs82aIV, IM845: rpm1(ur299)V; zdIs5I, IM817: rpm-1(ur299)V; slt-1(eh15)X; zdIs5I, IM841: clec-38(ur280)V; rpm-1(ur299)V; zdIs5I, IM828: clec-38(ur280)V; rpm1(ur299)V; slt-1(eh15)X; zdIs5I, IM868: clec-38(ur280)V; rpm1(ur299)V; unc-40(e1430)I; zdIs5I, IM823: urEx333 [mec-4::clec-38; flp-20::gfp]; clec-38(ur280)V, IM829: urEx 333 [mec4::clec-38; flp-20]; slt1(eh15)X; clec-38(ur280)V, IM882: evEx66[unc-40::GFP;rol-6]; clec38(ur280)V, IM981: clec-38(ur280)V;evIs98[unc-5::gfp;+dpy-20], IM982: clec 38(ur280)V;kyEx253[sax-3::gfp;+lin-15], IM977: urEx375[pclec-38::gfp; str-1::gfp], IM898: clec-38(ur280)V;kyEx1212[punc-86::UNC-40::GFP], IM995: clec-38(ur280)V;juIs1 [punc-25::SNB-1::GFP]IV.

Transgenes maintained as extrachromosomal arrays included the following: urEx333[mec-4::clec-38; flp-20::gfp], urEx375[pclec-38::gfp; str-1::gfp]. Strains not derived in the Wadsworth Laboratory were kindly provided by the Caenorhabditis Genetics Center (Minneapolis, MN) (NM1455, NL4256, CB4856, and other strains used for mapping), Scott Clark (SK4005; New York University Medical Center, New York, NY), Joe Culotti (evIs82a[punc-129::GFP], evEx66, evIs98; University of Toronto, Toronto, Ontario, Canada), Cori Bargmann (kyEx1212[punc-86::UNC-40::GFP]; The Rockefeller University, New York, NY), and Yishi Jin (juIs-1[punc-25::SNB-1::GFP]; University of California, San Diego, La Jolla, CA). The tm2035 deletion allele was obtained from the Japanese Knock-out Consortium as described by the National BioResource Project (http://www.nbrp.jp/report/reportProject.jsp? project $=$ celegans).

The tm2035 deletion was detected using the following PCR primers: forward, CGTTACAAAACCGTTGAG, and reverse, TAGTTGTTGATGCAATTC; and a 992 bp deletion was confirmed by sequencing. The clec-38(RNAi) phenotype was observed by feeding worms with bacteria expressing double-stranded RNA (Fraser et al., 2000) using the RNA interference (RNAi) feeding bacterial strain V-11O15 (Geneservice, Cambridge, UK). All double or triple mutants generated were confirmed by complementation tests or by PCR genotyping.

Molecular characterization of clec-38(ur280). Two factor and SNP mapping were used to place the ur280 mutation on the right arm of chromosome V. Cosmids containing sequences for the region were obtained from The Sanger Institute (Cambridge, UK) and were injected into mutant animals at concentrations of $2 \mathrm{ng} / \mu \mathrm{l}$, along with a coinjection marker pIM175 [Punc-119::gfp] at $80 \mathrm{ng} / \mu \mathrm{l}$. The animals were examined for rescue of a mild dumpy body phenotype caused by ur 280 mutation. Germline transformation with the single cosmid T25E12 rescued the phenotypes of $u r 280$. Furthermore, a genomic PCR product that included the entire sequence of the gene T25E12.10 (i.e., clec-38) rescued the ur280 phenotype. The molecular lesion in clec-38(ur280) was identified by sequencing genomic PCR products from mutant animals and aligning with the reported genomic sequence from C. elegans Genome Sequencing Consortium.

Plasmid construction. The mec-4::clec-38 expression construct pIM218 was made by amplifying clec- 38 sequence from a C. elegans cDNA library
(Invitrogen, Carlsbad, CA) using the following PCR primers: forward, CTACTAGCTAGCATGGCAATATTCTACGAC, and reverse, CGGCGGGGTACCTCAAAAATCAATAGCCCG. The PCR product was digested with NheI and KpnI restriction enzymes and was cloned into the NheI and KpnI sites after the mec-4 promoter sequence of plasmid pIM 207 (Quinn et al., 2006). The promoter fusion (Pclec-38::gfp) construct pIM220 was made by amplifying $\sim 2 \mathrm{~kb}$ sequence upstream of the $\mathrm{clec}-38$ (T25E12.10) ATG codon including the first three codons from C. elegans genomic DNA using the following PCR primers: forward, AAAACTGCAGAGAGGGGAATTTTCAAG, and reverse, CGCCGCGGATCCTATTGCCATTTGTTTTGC. The PCR product was digested with Pst I and $B a m \mathrm{H} 1$ restriction enzymes and was cloned into the Pst I and Bam $\mathrm{H} 1$ sites in the Fire laboratory vector, pPD95.77 (Mello and Fire, 1995).

Germline transformation. Transgenic strains were generated by described methods (Mello and Fire, 1995). The pIM218 construct was injected into clec-38(ur280) animals at $50 \mathrm{ng} / \mu \mathrm{l}$ along with a flp-20::gfp coinjection marker [kindly provided by Chris Li (City College of New York, New York, NY)] at $80 \mathrm{ng} / \mu \mathrm{l}$. The transgenic lines were maintained as extrachromosomal arrays by following the green fluorescent protein (GFP) fluorescence. Three independent lines were established. The array of one line, IM823, was used in genetic crosses with slt-1(eh15) to generate strain IM829. The pIM 220 construct was injected into N2 animals at $5 \mathrm{ng} / \mu \mathrm{l}$ along with $s t r-1:: g f p$ coinjection marker [a kind gift from Cori Bargmann (Rockefeller University, New York, NY)] at $80 \mathrm{ng} / \mu \mathrm{l}$. The transgenic lines were maintained as extrachromosomal arrays by following the GFP fluorescence. Three independent lines were established and analyzed for $\mathrm{clec}$-38 expression.

Microscopy and axon guidance assay. Mechanosensory neurons were visualized using a chromosomally integrated mec-4::gfp transgene ( $z$ dIs5; gift from Scott Clark), and the DA and DB neurons were observed using an integrated unc-129::gfp transgene evIs82a (a gift from J. Culotti). The animals were mounted on $5 \%$ agarose pad with $10 \mathrm{~mm}$ levamisole and were analyzed using a Carl Zeiss (Oberkochen, Germany) Axio-imager Z1 microscope with an apotome imager. The ventral guidance of the AVM axon was scored as defective if it failed to reach the ventral cord. Dorsal guidance of the DA and DB axons was scored as defective if none of the axons from the neuron cell bodies situated along the ventral nerve cord in the region between the pharynx and vulva failed to reach the dorsal cord. The PLM axon was scored as overextended if it migrated past the AVM neuron cell body. UNC-40::GFP expression analysis was performed using $63 \times$ objective in the wild-type and mutant background under same exposure time. Statistical analysis was done using a twotailed $z$ test to compare the phenotypes between two different strains. A value of $p<0.05$ was considered significantly different. Image analysis was performed using Axio-Vision LE 4.5 software.

\section{Results}

\section{Identification and characterization of CLEC-38}

A mutation, ur280, was isolated from a genetic screen for mutations that could suppress dorsal guidance defects caused by the unc-6(rh46) mutation. The unc-6(rh46) mutation is a partial lossof-function allele (Hedgecock et al., 1990; Wadsworth et al., 1996) and mutations were isolated that could improve the ability of DA and DB motor axons to reach the dorsal cord in the unc6(rh46) background. The ur280 mutation was mapped to a region of chromosome V (supplemental Fig. 1, available at www. jneurosci.org as supplemental material). Using cosmids containing sequence corresponding to this region and DNA-mediated transformation rescue, we found that the $u r 280$ locus is within cosmid T25E12 in the gene lec-38. The clec-38 gene is predicted to encode a protein of 382 aa comprising a short cytoplasmic tail with a putative $\mathrm{SH} 2$ binding site, a type-II transmembrane domain and two extracellular CTLDs (supplemental Fig. 1, available at www.jneurosci.org as supplemental material). DNA sequence analysis of ur280 identifies a TTT to GTT change, which causes valine 269 within the second CTLD to be substituted for phenylalanine. We also analyzed $t m 2035$, which was isolated in a PCR- 
based screen for deletions by the National BioResource Project. This clec-38 mutation can also suppress the dorsal guidance defects of unc-6(rh46) (data not shown). The tm2035 mutation is a deletion removing part of the $\mathrm{clec}-38$ promoter and the first five exons, which correspond to the first 222 aa of CLEC-38 (supplemental Fig. 1, available at www.jneurosci.org as supplemental material). Animals deficient for clec-38 by RNAi show a mild dumpy phenotype similar to that caused by the clec-38(ur280) and clec-38(tm2035) mutations. Furthermore, the ur280 phenotypes were not enhanced when the $u r 280$ allele was placed over the deficiency $y D f 4$, which removes the clec-38 gene (data not shown). Together, these observations indicate that clec-38(ur280) is a strong loss-of-function mutation.

To determine cells that express the clec- 38 gene, we designed a promoter fusion construct using $2 \mathrm{~kb}$ upstream of clec-38 sequence fused to a green fluorescent protein coding sequence (see Materials and Methods). The transgenic animals carrying this construct show GFP fluorescence in the nervous system (Fig. 1). Fluorescence could be detected in early embryos and persists through adulthood. The expression was detected in head neurons of threefold stage embryos (Fig. 1A). Strong expression was also observed in intestine. In the adults, the fluorescence is detected in the ventral cord motor neurons, PLM touch neurons, head neurons, and the intestine (Fig. $1 B, C$ ). Attempts to generate strains expressing a GFP-tagged CLEC-38 translational fusion have been unsuccessful.

\section{CLEC-38 regulates dorsal axon guidance mediated by UNC-40}

The clec-38(ur280) allele was isolated as a suppressor of DA and DB motor axon defects caused by the unc-6(rh46) allele. We examined whether the dorsal migration of DA and DB motor neuron axons in different unc- 6 mutants are improved by the clec38(ur280) mutation (Fig. 2). We found that dorsal guidance is improved in unc-6(rh46) and unc-6(e78) mutants, but not in unc-6(ev400) mutants (Fig. 2 F). The unc-6(rh46) and unc-6(e78) are reduction-of-function missense mutations that are predicted to encode altered forms of UNC-6, whereas unc-6(ev400) causes an early stop mutation and is considered a null allele (Hedgecock et al., 1990; Wadsworth et al., 1996). These results suggest UNC-6 is required for the improved guidance in the loss of clec-38 function mutant.

The dorsal guidance of DA and DB motor neurons in response to UNC-6 is mediated by UNC-5 and UNC-40 receptors (LeungHagesteijn et al., 1992; Chan et al., 1996). To determine whether the improved dorsal guidance observed in the clec-38(ur280) mutants are affected by loss of the receptors, we examined animals with $u n c-5$ and $u n c-40$ loss-of-function mutations (Fig. $2 \mathrm{~F}$ ). Loss of $u n c-5$, but not $u n c-40$, function causes dorsal axon guidance defective by the criteria of our assay, which scored dorsal migration as defective only if none of the axons from the neuron cell bodies situated along the ventral nerve cord in the region between the pharynx and vulva failed to reach the dorsal cord. Results from clec-38; unc-40; unc-6(rh46) and clec-38; unc-5; unc-6(rh46) triple mutants show that the clec-38 mutation cannot suppress the unc-6(rh46) dorsal guidance phenotype in the absence of UNC-40, indicating that the suppression by loss of clec-38 function requires UNC-40 signaling. The dorsal guidance defects are the same in clec-38; unc-5(e53); unc-6(rh46) and unc-5(e53) single mutants; however, this result is not too informative because dorsal guidance is severely impaired by the loss of unc- 5 function alone. Together, the results suggest that the loss of clec-38 func-
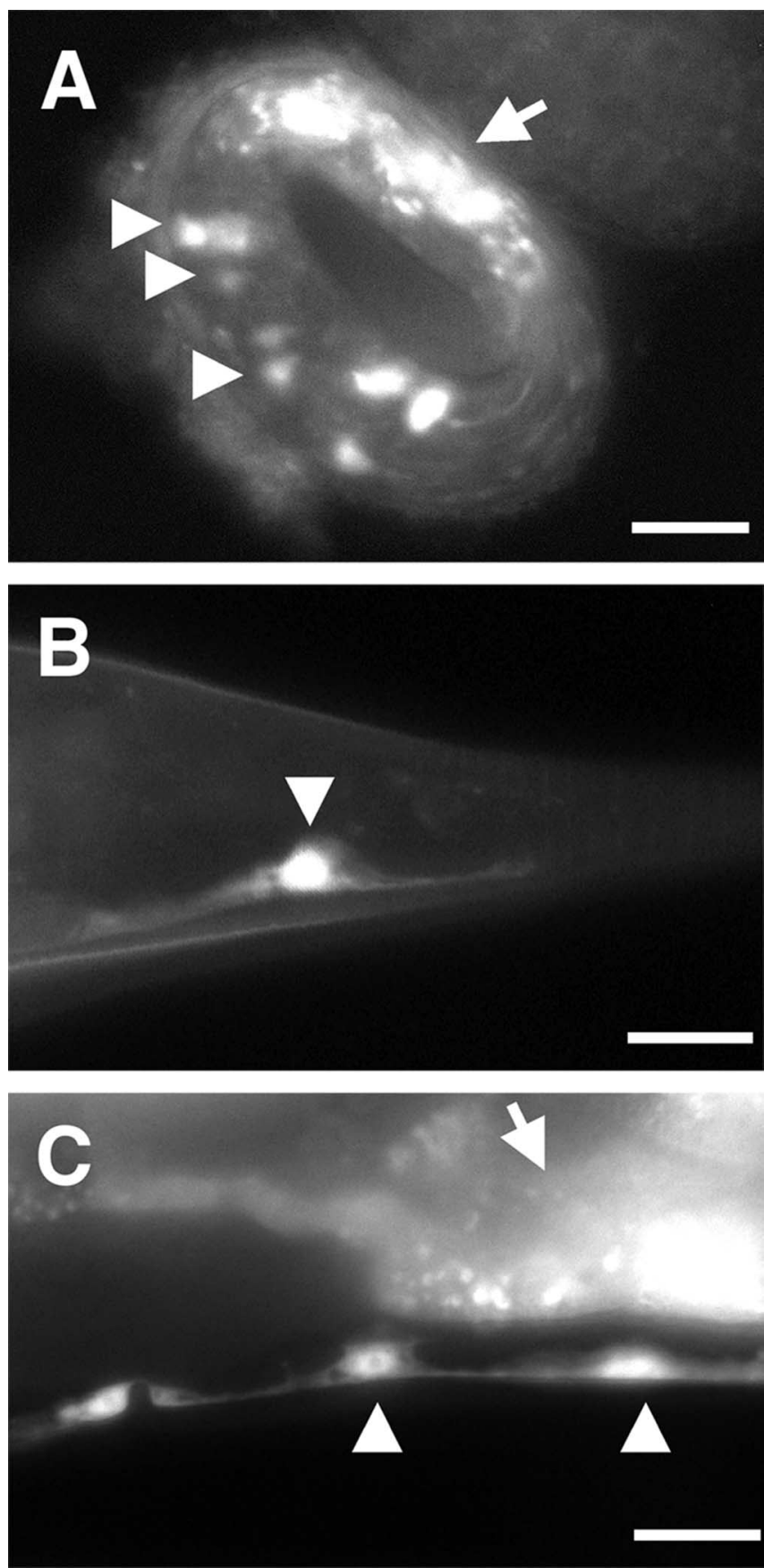

Figure 1. Expression of clec-38. Pattern of clec-38 promoter expression was obtained from an extrachromosomal array containing $2 \mathrm{~kb}$ upstream sequence of clec-38 fused with GFP. Expression is observed in a subset of neurons and in the intestine. Fluorescence could be detected in early embryos and persists through adulthood. $A$, Threefold stage embryo showing clec-38 expression in head neurons (arrowheads) and intestine (arrow). $\boldsymbol{B}$, Adult animal showing fluorescence in PLM neuron (arrowhead). $C$, Expression is detected in ventral cord motor neurons (arrowheads) and the intestine (arrow) in an adult. Anterior is to the left in $\boldsymbol{B}$ and $\boldsymbol{C}$. Scale bars, $20 \mu \mathrm{m}$.

tion affects UNC-40 activity in the DA and DB motor neurons and that this change improves the ability of the axons to respond to the dorsal guidance information provided by UNC-6.

\section{CLEC-38 regulates ventral guidance mediated by UNC-40}

The ventral migration of the AVM axon is guided by the UNC- 6 and SLT-1 guidance cues through signals mediated by 
the UNC-40 and SAX-3 receptors (Hedgecock et al., 1990; Wadsworth et al., 1996; Hao et al., 2001; Yu et al., 2002; Gitai et al., 2003). The AVM axon travels away from dorsal sources of SLT-1 and toward ventral sources of UNC-6 (Fig. $3)$. Loss of both cues results in almost complete failure of AVM axon ventral guidance, whereas the loss of either slt-1 or unc- 6 function results in $\sim 40 \%$ failure (Fig. $3 D-F)$. There is no significant difference in the penetrance of the defect between unc-6(ev400) and clec-38;unc$6($ ev400) double mutants or between unc-6(rh46) and clec-38;unc-6(rh46) double mutants (Fig. 3F). However, the ventral guidance defect observed in slt-1 mutants is suppressed in clec-38; slt-1 double mutants. These results suggest that loss of clec-38 function can improve the ability of UNC-6 to provide ventral guidance information. Consistent with the finding that UNC-40 is required for improving the dorsal guidance of the DA and DB motor neuron axons, the loss of clec-38 function could similarly improve the AVM response to UNC- 6 by enhancing UNC-40 activity. The ability of clec-38(ur280) to improve the ventral AVM axon guidance in the slt-1 loss-offunction background further indicates that the effect of the clec-38 mutation is not dependent on altered forms of UNC- 6 but can also improve guidance by the wild type UNC-6 protein.

We wanted to determine whether clec-38 expression by AVM is important for the phenotype. When the clec-38 promoter was used to express GFP, we did not specifically detect expression in AVM (Fig. 1); however, mosaic or low-level expression by the transgene could cause AVM expression to be missed. To test whether $c l e c-38$ acts cell autonomously in AVM, we expressed a clec-38 transgene under the control of the mec- 4 promoter to drive expression in the touch neurons, which includes AVM. This expression was able to reverse the suppression of the AVM guidance defects caused by the clec-38 mutation in clec-38; slt-1double mutants (Fig. $3 F$ ). This indicates that AVM expression of $c l e c-38$ can account for the axon guidance phenotypes.

\section{CLEC-38 is required for presynaptic terminal organization}

From the same genetic screen used to isolate the clec-38(ur280) mutation, we isolated an allele of $r p m-1$, which encodes a protein that regulates presynaptic terminal (Schaefer et al., 2000; Zhen et al., 2000). To determine whether mutations in $c l e c-38$ also disrupt presynaptic terminals, we analyzed the presynaptic terminals of the GABAergic DD and VD motor neurons using a transgene expressing synaptobrevin

A

$\mathbf{F}$ Scale bars, $20 \mu \mathrm{m}$.
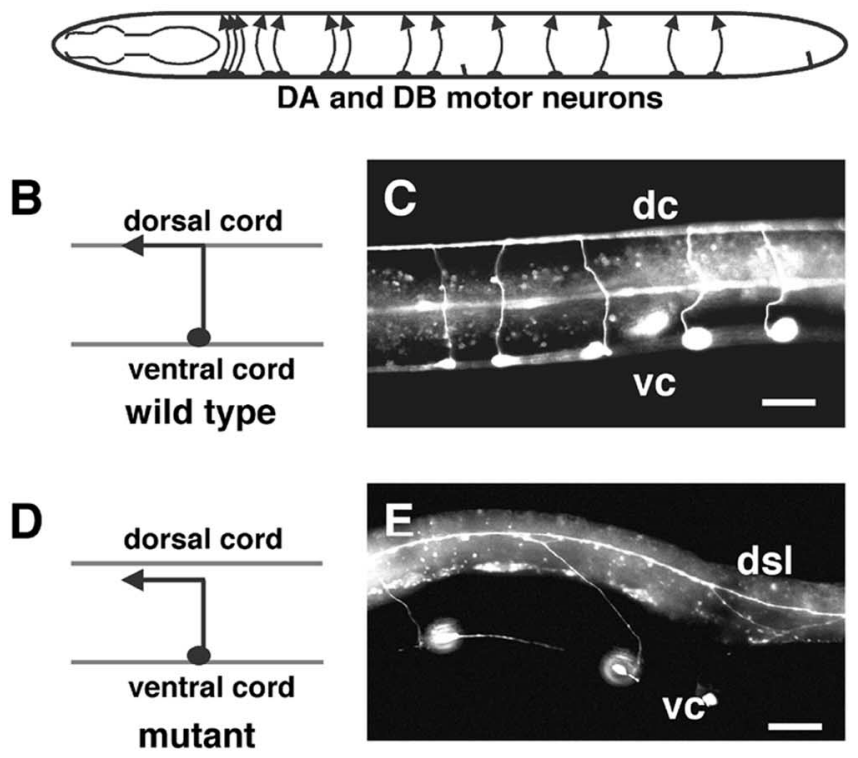

$n=350 \quad n=450 \quad n=490 \quad n=257 \quad n=340 \quad n=405 \quad n=380 \quad n=200 \quad n=260 \quad n=260 \quad n=390$

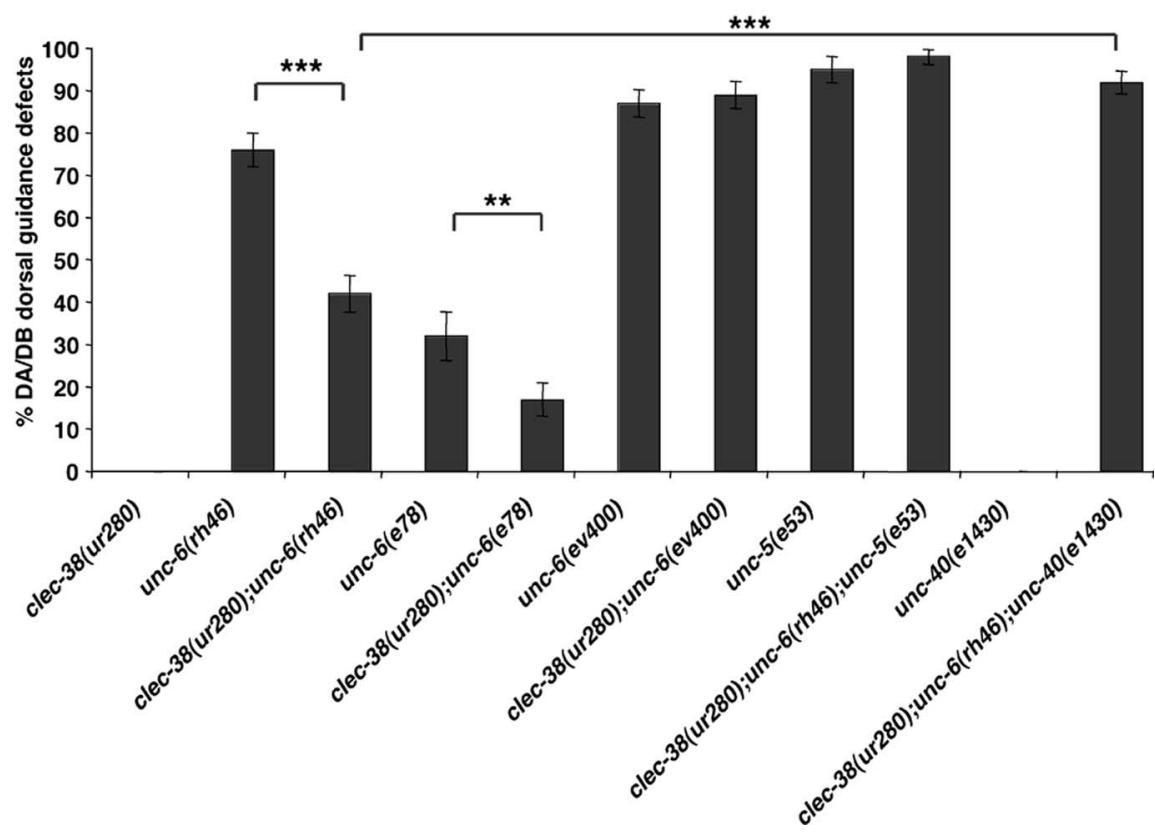

Figure 2. clec-38 affects unc-6-dependent dorsal guidance. $A$, Schematic diagram of the migration of DA and DB motor axons. These axons migrate away from ventral UNC-6 sources. $B-E, D A$ and $D B$ axon guidance in wild-type and mutant $L 4$ larva. DA and DB axons in L4 stage animals were visualized with evls82a [unc-129::gfp] in wild-type $(\boldsymbol{C})$ and unc-6(rh46) $(\boldsymbol{E})$ animals. Axons between the pharynx and vulva were observed, and dorsal guidance was scored as defective if all the axons failed to reach the dorsal cord. In this image, axons have only extended as far as the dorsal sublateral nerve. $F$, clec-38(ur280) suppresses the dorsal guidance defects caused by the unc-6(rh46) and unc-6(e78) partial loss-of-function alleles but not the null allele, unc-6(ev400). The suppression of dorsal guidance defects in clec-38(ur280); unc-6(rh46) is inhibited by the loss of unc-40 function, as observed in the triple mutant, clec-38(ur280);unc-6(rh46); unc-40(e1430). This suggests that the suppressor activity of clec-38(ur280) is dependent on unc-40 function. Error bars represent SEs of proportions, and asterisks indicate statistically significant difference $\left({ }^{* *} p<0.005 ;{ }^{* * *} p<0.0005\right)$. Anterior is to the left, and dorsal is up. vc, Ventral cord; dc, dorsal cord; dsl, dorsal-sublateral cord.

fused to GFP (SNB-1::GFP) and driven by the unc-25 promoter (Hallam and Jin, 1998; Nonet, 1999).

In wild-type animals, SNB-1::GFP puncta are uniform in shape and are evenly distributed along the processes (Fig. 4A,B). 
A

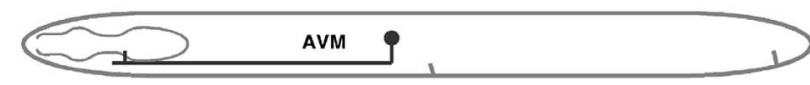

B

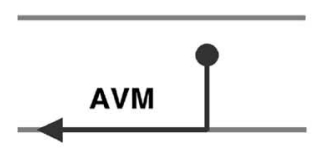

wild type

D

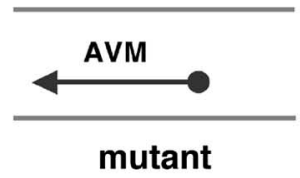

$\mathbf{F}$

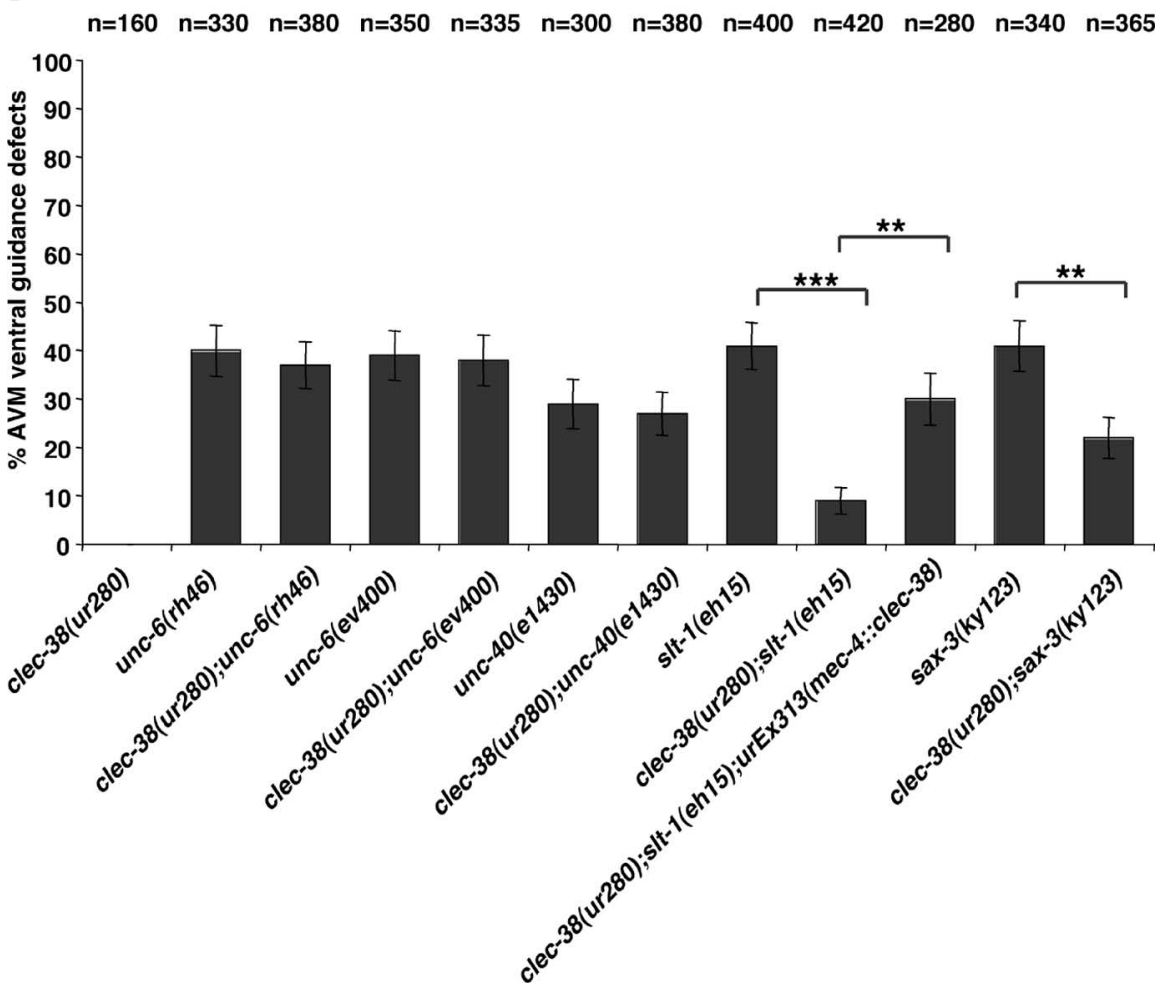

Figure 3. CLEC-38 and ventral guidance mediated by UNC-40. $A$, Schematic diagram of the AVM axon. The AVM axon is repelled from dorsal SLT-1 sources and is attracted toward ventral UNC-6 sources. $\boldsymbol{B}-\boldsymbol{E}$, AVM axon guidance in wild-type and mutant larva. The AVM axons in L4 stage animals were visualized with zdls5 [mec-4::gfp] in wild-type ( $\boldsymbol{C}$ ) and mutant (E) animals. $\boldsymbol{F}$, The AVM guidance defects caused by s/t- 1 and sax-3 loss-of-function mutations are suppressed by the loss of clec-38 function, suggesting enhancement of UNC-6 signaling is likely through improved UNC-40 activity. This effect is reversed when clec-38 is expressed in AVM, suggesting that clec-38 acts cell autonomously. Error bars represent SEs of proportions, and asterisks indicate statistically significant difference $\left({ }^{* *} p<0.005 ;{ }^{* *} p<0.0005\right)$. Anterior is to the left, and dorsal is up. Scale bars, $20 \mu \mathrm{m}$.

axons (data not shown), suggesting that axon guidance defects are not the cause of the irregular SNB-1::GFP pattern in clec38(ur280) mutants.

clec-38 genetically interacts with rpm-1, which encodes a presynaptic terminal protein

RPM-1 is a large protein localized to the presynaptic periactive zone in the mature nervous system. Loss of rpm-1 function causes a range of defects affecting neuronal morphology and synaptic organization (Schaefer et al., 2000; Zhen et al., 2000; Nakata et al., 2005). In both clec-38 and rpm-1 mutants similar GABAergic motor neuron synapse defects are observed. In addition, loss of $r p m-1$ function similarly affects axon outgrowth mediated by guidance receptors, although RPM-1 negatively regulates the UNC-5 and SAX-3 receptors rather than UNC-40 (Li et al., 2008). Furthermore, RPM-1 appears to regulate the UNC-5 and SAX-3 receptors through a pathway involving the Rab GEF GLO-4; however, the guidance phenotypes of glo-4 and $\mathrm{clec}-38$ mutations are different, suggesting that $c l e c-38$ do not act through this pathway.

To further explore a relationship between RPM-1 and CLEC-38 functions, we determined whether there are genetic interactions between $\mathrm{clec}-38$ and rpm-1. For the DA and DB motor neurons, the axon guidance phenotype caused by unc-6(rh46) is suppressed by both clec-38 and rpm-1 mutations (Fig. 5A) (Li et al., 2008). In clec-38; rpm-1; unc-6(rh46) triple mutants, the penetrance is similar to mutants with $u n c$ 6(rh46) alone (Fig. 5A). Thus, the loss of clec-38 function reverses the effects of the loss of $r p m-1$. For the ventral migration of the AVM axon, we find a $90 \%$ penetrant defect in the rpm-1;slt-1double mutants, but only a $21 \%$ penetrant defect in rpm-1;slt-1; clec-38 mutants (Fig. 5B). Again, the loss of clec-38 function reverses the effects of the loss of rpm-1 function.

We also observe that double mutations have an effect on the control of axon extension. In rpm-1 mutants, the PLM anterior process axon often extends beyond the normal termination point (Fig. 6A-E) (Schaefer et al., 2000; Li et al., 2008). We find that the overextension caused by the loss of rpm-1

In $86 \%$ of clec-38 mutants, the distribution of SNB-1::GFP puncta is irregular, leaving clear areas in the dorsal and ventral cord (Fig. 4C,D). The number of dorsal and ventral cord puncta is reduced in $\mathrm{clec}-38$ mutants (Fig. $4 E$ ). In addition, the remaining puncta are of irregular shape and size (Fig. $4 C, D$ ). To determine whether these synaptic defects could be a result of gross axon guidance defects, we checked the morphology of DD and VD axons in lec-38 mutants. We did not observe mispositioned function is affected by the loss of clec-38 function. Interestingly, the penetrance of the overextension phenotype in clec-38; rpm-1 mutants is not greater than that of $r p m$ - 1 mutants; rather, the length of the extension increases (Fig. 6G,H). Whereas in rpm-1(ur299) mutants only $15 \%$ of the PLM axons extend beyond the position of the AVM cell body, $83 \%$ of the axons in clec-38; rpm-1 mutants extend beyond the AVM cell body (Fig. $6 H$ ). This effect is suppressed by the loss of UNC-40 in unc-40; clec-38; rpm-1 triple mutants, suggesting 
that the additional extension is likely attributable to the effect on UNC-40 by loss of clec-38 function. The effect is also suppressed by expression of the $c l e c-38$ transgene under the control of the mec- 4 promoter, suggesting that clec-38 acts cell autonomously in the PLM touch neuron (data not shown).

\section{clec-38 regulates the $\mathrm{UNC}-40:: \mathrm{GFP}$} expression pattern

To further explore the relationship between UNC-40 and CLEC-38, we used transgenic strains expressing functional UNC-40::GFP. In the first case, we used a transgene that contains the $u n c-40$ promoter sequence to drive UNC-40::GFP expression (Chan et al., 1996). Although the construct used in making this transgenes may have an in-frame deletion of 40 aa in the third Ig domain of the protein (Joe Culotti, personal communication), it is able to rescue $u n c-40$ null mutant phenotypes (Chan et al., 1996). In this strain, the GFP signal is detectable in all cells during early gastrulation and then gradually decreases, with later expression observed in neurons and motile cells (Chan et al., 1996). We observe that $20 \%(n=50)$ of the clec$38(+)$ animals with UNC-40::GFP expression have neurons with abnormal morphologies, including multiple processes (Fig. 7). Excess axon outgrowth and the abnormal neuronal morphology is likely caused by higher levels of UNC-40 because similar phenotypes are associated with the expression of constitutively active forms of UNC-40 in neurons (Gitai et al., 2003).

Compared with the clec-38 $(+)$ animals, $80 \%(n=50)$ of mutants heterozygous for the $\mathrm{clec}-38$ mutation have severe morphological defects (Fig. 7) and 100\% $(n=50)$ of mutants homozygous for the clec-38 mutation die as embryos. In some individual neurons, differences in the intensity of the GFP signal were noticeable. For example, in ventral cord motor neurons, only weak expression was observed in $5 \%(n=50)$ of the clec-38 $(+)$ animals, whereas $45 \%(n=50)$ of clec-38 heterozygous mutants showed strong UNC-40::GFP expression (Fig. $7 A, B$ ). From these observations, we infer that high levels of UNC-40::GFP cause morphological changes and that loss of clec-38 activity influences the levels or cellular distribution of UNC-40. Analyses of UNC-5::GFP and SAX-3::GFP expression patterns in clec-38 mutants did not reveal major differences from the patterns seen in the wild-type background (data not shown).

We observe that overexpression of the above unc-40::gfp transgene causes severe morphological changes throughout the animal. However, because of the possible effects of the deletion within the $u n c-40$ sequence and because the identification of individual neurons is difficult in these strains (Fig. 7C,D), we also examined the effects of a different $u n c-40$ transgene that is expressed in fewer cells. We used a transgene that expresses UNC40:GFP under the control of the unc-86 promoter, which drives expression in a subset of cells, which include the HSN neuron (Adler et al., 2006) (Fig. 7E). We observe that expression of this transgene in clec-38 mutants results in strong UNC-40::GFP expression and HSN morphological changes, including multiple axon outgrowths (Fig. 7F). In 78\% of clec-38(ur280) mutants $(n=115)$, there is strong GFP signal in HSN compared with $25 \%$ $(n=105)$ in clec-38(+) animals. Furthermore, in $95 \%$ of the mutants, the HSN cell morphology is abnormal and has excessive axon outgrowth. These morphological defects are observed in all larval stages (data not shown). Together, these results suggest that loss of clec-38 function enhances UNC-40::GFP expression. This is consistent with the genetic results that indicate clec-38 negatively regulates UNC-40 activity.

\section{Discussion}

Proteins with C-type lectin-like domains can regulate axon guidance and synaptogenesis

Our results indicate that CLEC-38 regulates axon outgrowth and presynaptic patterning. The ur280 loss-of-function allele has a missense mutation within the second CTLD, suggesting that these CLEC-38 functions are dependent on the activity mediated by this domain. To our knowledge, members of the protein superfamily that contain the CTLD have not been implicated before in axon guidance or synaptogenesis. Indeed, little is known about the function of members of this family in C. elegans, despite the fact that it is considered the second largest family of proteins in C. elegans (Drickamer and Dodd, 1999; Dodd and Drickamer, 2001). The CTLD is distinguished by sequence similarity; it is found in a variety of vertebrate and invertebrate proteins and it is known to mediate binding to carbohydrates, proteins, and inorganic molecules (Dricka- 
A

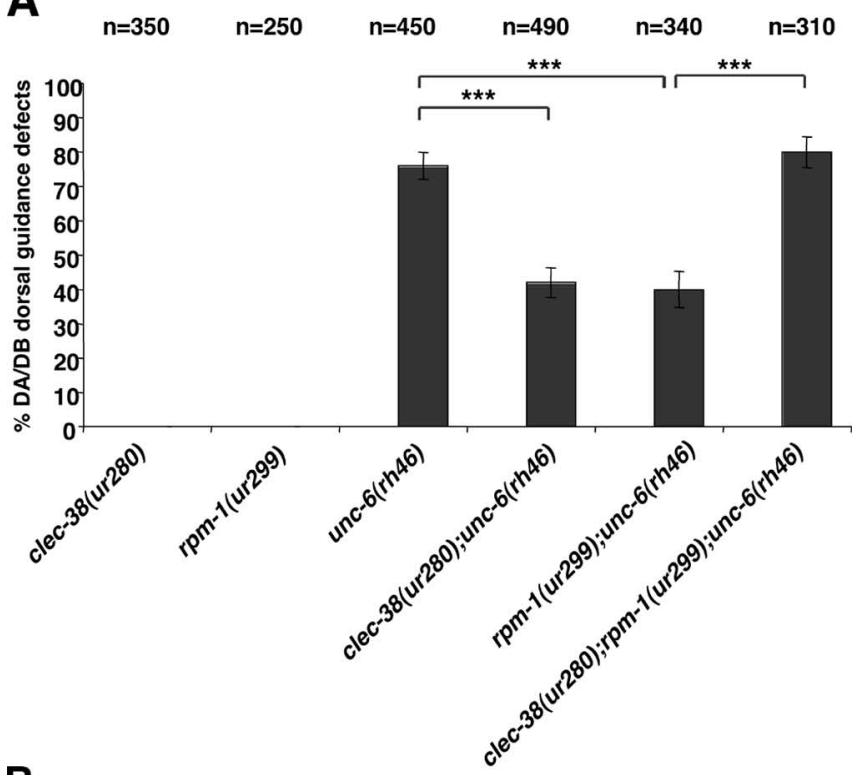

B $n=160 \quad n=200 \quad n=250 \quad n=400 \quad n=180 \quad n=300 \quad n=310$

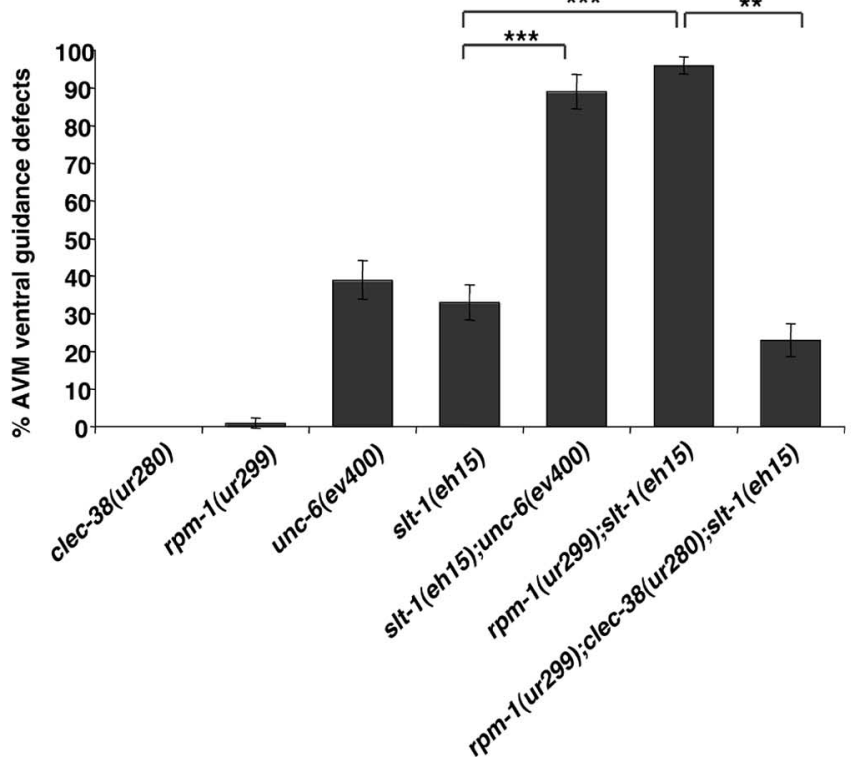

Figure 5. CLEC-38 regulates UNC-40 and RPM-1-dependent axon outgrowth. $A$, The DA and DB motor neuron dorsal guidance defects caused by the unc-6(rh46) mutation are partially suppressed by either the clec38(ur280) or the rpm-1(ur299) mutation. However, this effect is reversed in the triple mutant, clec-38(ur280);rpm-1 (ur299); unc-6(rh46), suggesting that the suppressor activity of both mutations is dependent on each other. $\boldsymbol{B}$, The rpm-1;slt-1 double mutants are as severe as s/t-1; unc- 6 mutants, suggesting that loss of rpm- 1 function prevents UNC- 6 signaling, likely by inhibiting UNC-40 signaling. This silencing effect is dependent on the SAX-3 receptor (Li et al., 2008). The silencing effect observed in rpm-1; 1 lt- 1 mutants is reversed in the triple clec-38;rpm-1; s/t-1 mutants, suggesting that the increased UNC-40 activity by loss of clec-38 function reverses the inhibition caused by the rpm- 1 mutation. Error bars indicate SE of proportions, and asterisks indicate statistically significant difference $\left({ }^{* *} p<0.005\right.$; ${ }^{* * *} p<$ $0.0005)$.

mer, 1999; Zelensky and Gready, 2003, 2005). Vertebrate family members have been implicated in functions such as extracellular matrix structure, endocytosis, complement activation, pathogen recognition, and cell-cell interactions. The diversity of this superfamily arises because CTLDs are found with a wide variety of other structural modules.
A

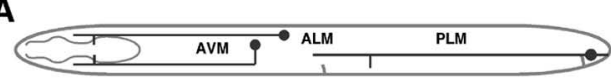

B

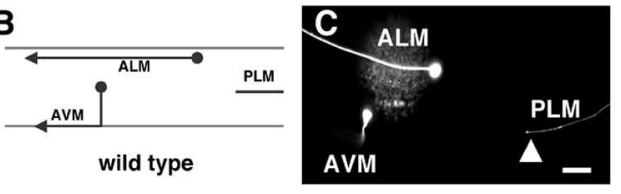

D

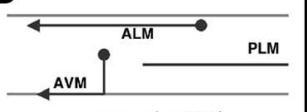

rpm-1(ur299)
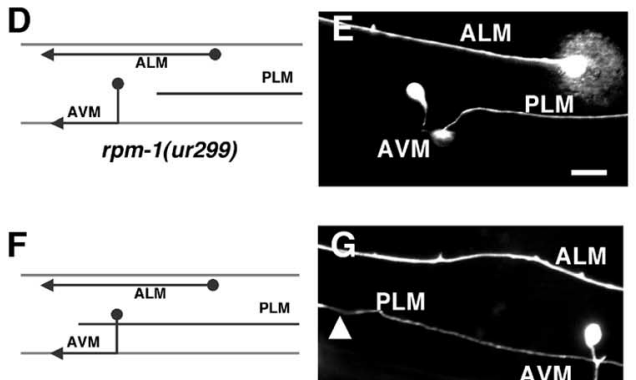

rpm-1(ur299);clec-38(ur280)

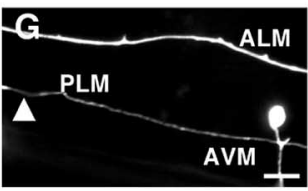

H

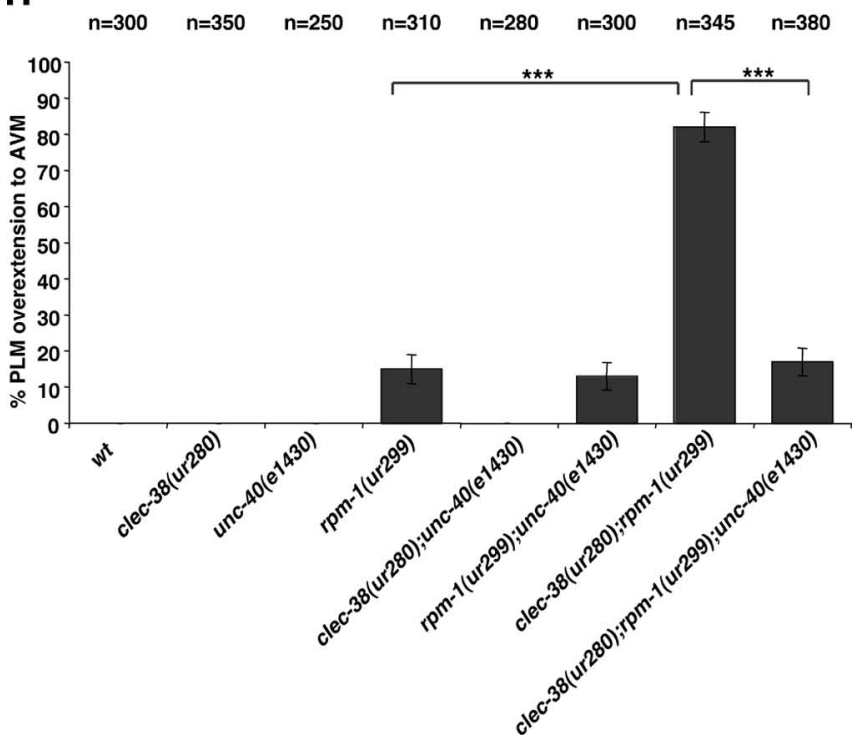

Figure 6. CLEC-38 and RPM-1-dependent PLM axon outgrowth. Loss of clec-38 function can affect PLM axon outgrowth. $A$, Schematic diagram of the PLM longitudinal axon. $B, C$, In wildtype animals, the PLM extend to a position near the vulva. $\boldsymbol{D}, \boldsymbol{E}$, In rpm-1 mutants, the PLM axon overextends, passing the ALM cell body. $F, G$, In clec-38;rpm- 1 mutants, the PLM axon extends beyond the AVM cell body position. The neurons were visualized with zdls5 [mec-4:.gfp] in $\boldsymbol{C}, \boldsymbol{E}$, and $\boldsymbol{G}$. $\boldsymbol{H}$, Loss of rpm-1 function causes overextension of PLM axon. Loss of both clec-38 and rpm-1 function cause longer extension of PLM, which crosses the AVM. This phenotype is dependent on unc- 40 because the triple mutant, clec-38,rpm-1; unc-40, phenocopies the rpm-1 single mutant. Error bars indicate SE of proportions, and asterisks indicate statistically significant difference $\left({ }^{* *} p<0.0005\right)$. Anterior is to the left, and dorsal is up. Scale bars, $20 \mu \mathrm{m}$.

\section{CLEC-38 negatively regulates UNC-40 axon}

outgrowth-promoting activity

We present genetic evidence that CLEC-38 negatively regulates UNC-40-mediated axon guidance activity. The enhancement of the dorsal guidance of DA and DB motor neuron axons in unc6(rh46) mutants by loss of clec-38 function is suppressed by the loss of $u n c-40$ function, suggesting that in the clec-38 mutant UNC-40 activity is enhanced and that this improves the ability of the axons to migrate dorsally in response to the unc-6(rh46) product. Furthermore, the ability of clec-38(ur280) to improve the ventral AVM axon guidance in the slt-1 loss-of-function 

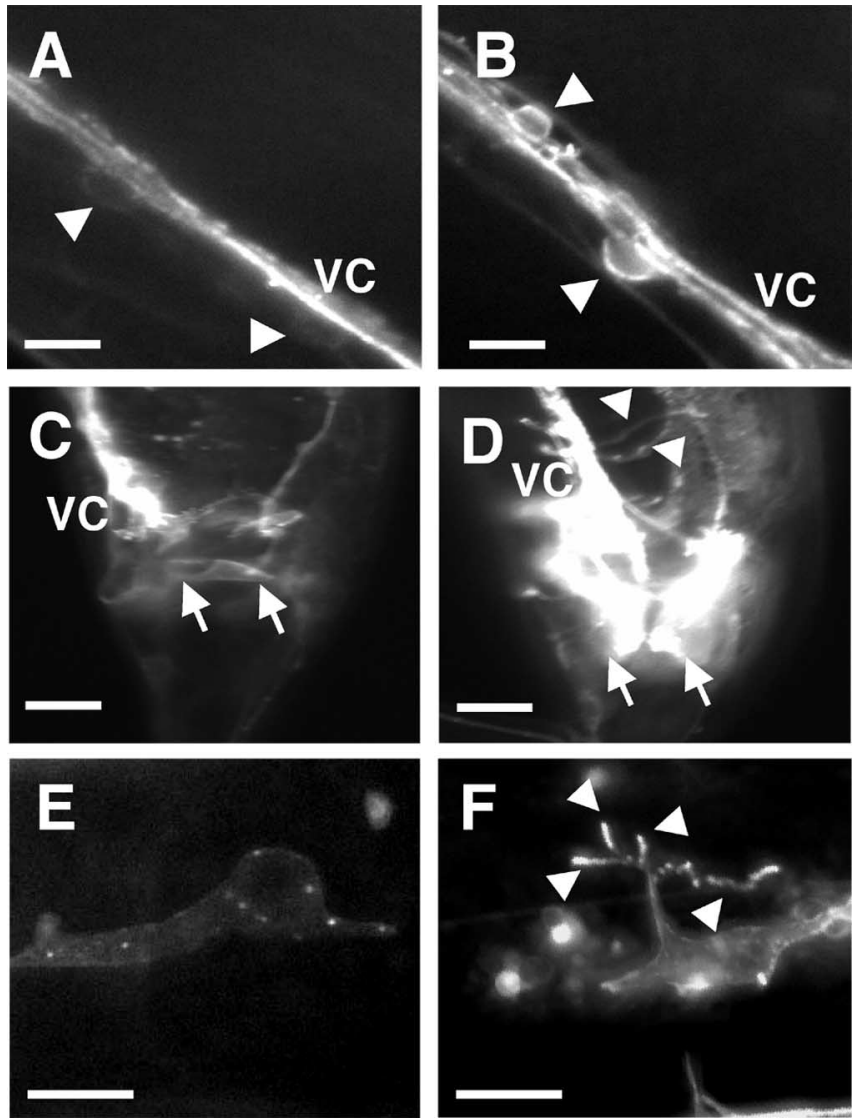

Figure 7. CLEC-38 affects UNC-40 expression. $\boldsymbol{A}, \boldsymbol{B}$, Ventral cord (vc) motor neurons of evEx66[unc-40::GFP] and clec-38(+/-);evEx66[unc-40::GFP] animals, respectively. Arrowheads indicate the neuronal cell bodies. UNC-40::GFP signal is weak in the wild-type neurons and is strong in clec-38(+/-) background. Anterior is to the left, and dorsal is up. C, D, Ventral cord (vc) and tail neurons (arrows) of evEx66[unc-40::GFP] and clec-38(+/-);evEx66[unc40::GFP] animals, respectively. Note a higher fluorescence signal is observed in clec-38(+/-) animals compared with wild-type animals. Also, in clec-38(+/-) animals, there are extra branches from ventral cord (arrowheads). Ventrolateral view with anterior to the top. $n=50$ for each group. Scale bars, $20 \mu \mathrm{m} . \boldsymbol{E}, \boldsymbol{F}, \mathrm{HSN}$ neuron of wild-type and clec-38 mutant animals expressing a transgene, kyEx1212[punc-86::UNC-40::GFP]. In wild-type HSN, UNC-40::GFP is localized to membranes at low level, whereas in clec-38 mutants, cell morphology is changed (arrow) with strong GFP signal and multipolar outgrowths (arrowheads). Shown is a lateral view with anterior on left. Scale bars, $10 \mu \mathrm{m}$.

background is consistent with the loss of clec-38 function enhancing the UNC-40-mediated ventral guidance response to UNC-6.

Although separate models can be made based on the phenotypes observed in each individual neuron type, here we discuss a model to explain phenotypes observed in all the neurons. We consider that the underlying guidance functions of CLEC-38 are the same in every neuron, although its function might be modified by the addition or absence of factors specific to a neuron. In this model, axon guidance is a multistep process with the axon guidance receptors involved at different steps (Fig. $8 A$ ). In the first step, the association of the ligand enables signaling that upregulates receptor levels and causes the asymmetric localization of receptors to the cell membrane where axon outgrowth will occur. In the second step, the guidance receptors promote axon outgrowth in the direction that was set up during the first step. At this step, the axon outgrowth-promoting activity of a receptor may act independently of its guidance cue ligand. As evidence for this, it was shown that either the attractive or repulsive cue, UNC$6 /$ netrin and Slt-1/slit, respectively, can suppress multipolar

\section{A. A model for guidance receptor signaling}

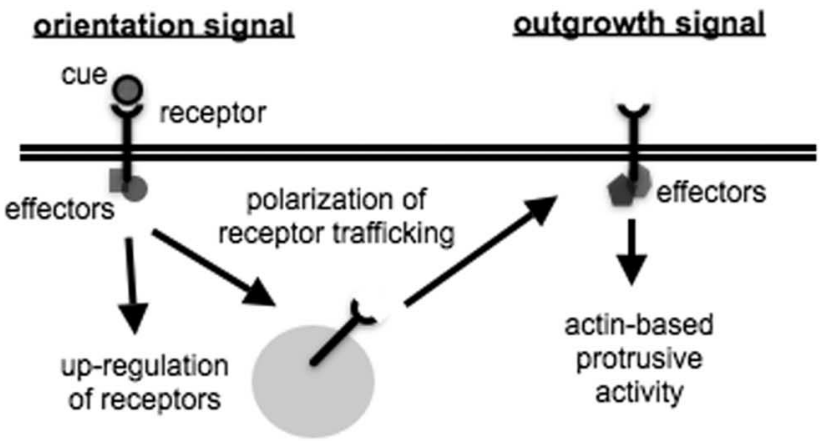

\section{B. A model for the role of CLEC-38 and RPM-1 in axon guidance and synaptogenesis}
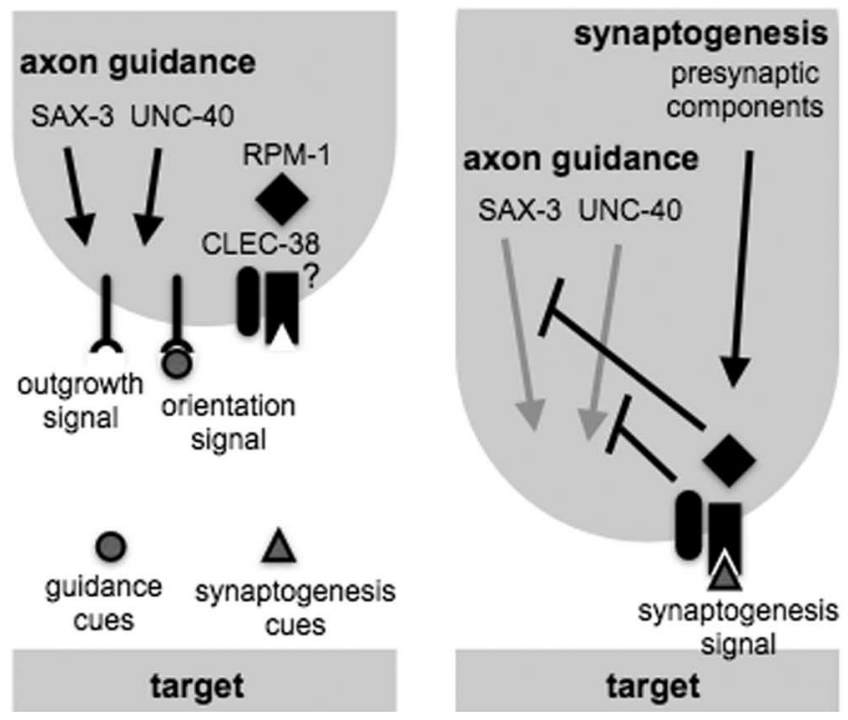

Figure 8. Model for guidance receptor activities and their regulation by CLEC-38 and RPM-1. $A$, It is proposed that the association of guidance cues with the guidance receptors signals an upregulation of guidance receptors and triggers polarity that causes the asymmetric trafficking of the receptors to specific cell membrane surfaces. The guidance receptors become part of protein complexes that promote actin-based protrusive activity. $\boldsymbol{B}$, At a distance (left panel), axon guidance cues predominate and stimulate outgrowth toward the target. However, as the migrating growth cone approaches its target (right panel), synaptogenesis cues trigger CLEC-38 and RPM- 1 activity in the neuron. CLEC-38 inhibits UNC-40, and RPM- 1 inhibits UNC -5 and SAX-3. In addition, CLEC-38 and RPM-1 regulate synapse formation. RPM-1 is localized to the presynaptic periactive zone in the mature nervous system (Nakata et al., 2005) and may regulate axon outgrowth and synaptogenesis by controlling vesicular trafficking through a GLO-4 Rab GTPase pathway (Grill et al., 2007; Li et al., 2008). The CLEC-38 sequence suggests a transmembrane protein with extracellular C-type lectin-like domains, which are known to mediate binding in other proteins. A mutation suggests that one of the C-type lectin-like domains is required for CLEC-38 function. The downstream signaling pathway that mediates negative regulation of UNC-40 is not clear.

AVM axon outgrowth caused by overexpression of the downstream effector MIG-10. In either case, there is enhanced ventral guidance of a single axon, indicating axon outgrowth-promoting activity that acts independently of the guidance cues can be oriented ventrally by either the attractive or repulsive cue (Quinn et al., 2006). Moreover, before the ventral outgrowth of the HSN axon, UNC-6 and UNC-40 trigger the ventral asymmetric localization of UNC-40 as well as proteins that promote actin-based protrusive activity (Adler et al., 2006; Chang et al., 2006; C. Quinn, D. Pfeil, and W. G. Wadsworth, unpublished observations). 
The results presented here indicate that $c l e c-38$ negatively regulates UNC-40 axon outgrowth-promoting activity rather than the orientation activity. The outgrowth-promoting activity affects the circumferential axon guidance, as well as longitudinal extension. We do not observe evidence of polarity defects in the clec-38 mutants, as might be expected if the orientation of the outgrowth was perturbed. For example, we observe that PLM overextension caused by loss of $r p m-1$ function is increased in the clec-38; rpm-1 double mutants and that the penetrance of this phenotype is reduced by the loss of $u n c-40$ function. This suggests that loss of clec-38 function enhances an UNC-40-mediated outgrowth activity for the longitudinal extension of PLM. Other genes have also been shown to affect UNC-40-mediated outgrowth in the anterior-posterior direction; however, in contrast to $c l e c-38$, these genes appear to affect neuronal polarity, with mutations causing inappropriate UNC-40 localization (LevyStrumpf and Culotti, 2007; Watari-Goshima et al., 2007). In these mutants, the UNC-40-mediated axon outgrowth activity may be normal, but misdirected.

An UNC-6-independent UNC-40 axon outgrowthpromoting activity also helps explain why, relative to the unc6(rh46) mutants, the axon guidance defects of the motor neuron axons, but not of the AVM axon, are suppressed in the clec38(ur280);unc-6(rh46) mutants. We hypothesize that the unc6(rh46) product, UNC-6 A157P, associates with UNC-40 but does not trigger signaling. Indeed, we have isolated an unc-40 allele that contains a missense mutation altering a single amino acid in the ectodomain sequence and that allows the UNC-40 mutant protein to orient axon outgrowth in unc-6(rh46) mutants (Z. Xu and W. G. Wadsworth, unpublished observations). For the motor neurons, UNC-6 A157P interacts with UNC-5 to polarize axon outgrowth dorsally. Upregulation of UNC-40 in the clec-38(ur280);unc-6(rh46) mutants allows some dorsal UNC40-mediated axon outgrowth-promoting activity, particularly because the motor neuron growth cones travel away from ventral UNC-6 A157P sources where it is less likely that UNC-40 activity will be inhibited by an association with UNC-6 A157P. For the AVM axon migration in the clec-38(ur280);unc-6(rh46) mutants, upregulation of UNC-40 does not have the same enhancement effect that it has with the motor neuron axons because the AVM axon migrates ventrally, toward the UNC-6 A157P sources, where it is more likely that UNC-40 activity will be inhibited by the association with UNC-6 A157P.

In addition to the axon orientation and outgrowthpromoting activities of the receptors, the results suggest that interactions between receptors can cause silencing. For the guidance of the AVM axon, loss of rpm-1 function in slt-1 mutants causes severe AVM ventral guidance defects, but the addition of the $c l e c-38$ mutation reverts the penetrance of the phenotype to that observed in mutants with the slt-1 mutation alone. We recently published evidence that loss of $r p m-1$ function enhances SAX-3 activity and that this increase in SAX-3 silences the ventral axon guidance mediated by UNC-40 (Li et al., 2008). While this manuscript was under review, a similar model predicting that SAX-3 could inhibit UNC-40-mediated guidance of the AVM axon was suggested (Fujisawa et al., 2007). These observations are consistent with in vitro analyses reporting that an association between the DCC and Robo receptors silences the ability of DCC to mediate turning toward netrin (Stein and Tessier-Lavigne, 2001). We now observe that enhancing UNC-40 activity can reverse the SAX-3 silencing of UNC-40.

Similarly, we propose that the composition of guidance receptors influences the dorsal guidance of the DA and DB motor neuron. Loss of clec-38 function enhances UNC-40 expression in neurons and improves dorsal guidance in the unc-6(rh46) mutant. Loss of $r p m-1$ function also improves dorsal guidance in the unc-6(rh46) mutant; however, unlike the clec-38 mutations, the rpm-1 mutations suppress the unc-6(rh46) dorsal guidance phenotype in the absence of UNC-40, indicating that the suppression by loss of $r p m-1$ function is not mediated through UNC-40 (Li et al., 2008). Instead, the loss of $r p m-1$ function enhances UNC-5 expression in the motor neurons (Li et al., 2008). Interestingly, combining the mutations in a clec-38; rpm-1; unc-6(rh46) triple mutant does not further enhance dorsal guidance; instead, the result is a guidance phenotype similar to mutants with unc6(rh46) alone. We propose loss of clec-38 function enhances UNC-40 outgrowth-promoting activity, but the upregulation of UNC-40 is not enough to alter UNC-5-mediated polarity effects, and therefore the direction of outgrowth remains dorsal. In the clec-38; rpm-1; unc-6(rh46) triple mutant, however, the enhanced expression of both UNC-5 and UNC-40 leads to the silencing of receptor activity, a situation that is similar to the proposed silencing by the association of SAX-3 and UNC-40 in AVM. There is evidence from experiments using cultured Xenopus spinal cord neurons that UNC-5 and DCC can physically interact, and that the expression of UNC5 in DCC-expressing neurons can convert the response to netrin from attractive to repulsive (Hong et al., 1999). This switch might occur if the introduction of UNC5 into the Xenopus neurons alters trafficking polarity enough to direct axon outgrowth activity toward the side opposite the netrin source. This may coincide with receptor complexes of UNC5 and DCC forming and silencing outgrowth activity.

Finally, consistent with the genetic evidence for the negative regulation of UNC-40-mediated activity by CLEC-38, we find that loss of $c l e c-38$ function affects the expression of transgenes encoding UNC-40::GFP. In the clec-38 mutant, morphological defects caused by transgene expression are enhanced. We interpret the more severe phenotypes to be a consequence of increased UNC-40 expression, in part because the expression of constitutively active forms of UNC-40 produce similar morphological defects (Gitai et al., 2003).

\section{A model for CLEC-38 function}

Our results indicate that CLEC-38 negatively regulates UNC-40mediated axon guidance as well as promotes the organization of presynaptic terminals. What is the connection between axon guidance, synaptogenesis, and the functions of clec-38 and rpm-1? An attractive idea is that CLEC-38 and RPM-1 are part of a process that regulates axon outgrowth in response to signals that promote synaptogenesis (Fig. $8 \mathrm{~B}$ ). As a growth cone reaches a target, cues from the target induce CLEC-38 and RPM- 1 activity that inhibits the guidance receptors and helps promote the formation of presynaptic structures. CLEC-38 negatively regulates UNC-40, whereas RPM-1 negatively regulates UNC-5 and SAX-3. Our results with double clec-38 and rpm-1 mutants indicate a complex relationship between CLEC-38 and RPM-1 with regards to their guidance functions; the loss of function of one molecule can switch the effects caused by the loss of function of the other molecule. As discussed above, we suggest that this relationship could be attributable to the silencing of receptor activities through the formation of receptor complexes comprising UNC-40 with UNC-5 or SAX-3. However, other models are possible, including more direct regulatory interactions between CLEC-38 and RPM-1. In any case, the results indicate distinct roles for CLEC-38 and RPM-1 in regulating the guidance process, and they suggest interactions that are part of a versatile system to 
regulate morphology changes of growth cones as they reach their targets and make connections.

\section{References}

Adler CE, Fetter RD, Bargmann CI (2006) UNC-6/Netrin induces neuronal asymmetry and defines the site of axon formation. Nat Neurosci 9:511-518.

Brenner S (1974) The genetics of Caenorhabditis elegans. Genetics 77:71-94. Chan SS, Zheng H, Su MW, Wilk R, Killeen MT, Hedgecock EM, Culotti JG (1996) UNC-40, a C. elegans homolog of DCC (Deleted in Colorectal Cancer), is required in motile cells responding to UNC-6 netrin cues. Cell 87:187-195.

Chang C, Adler CE, Krause M, Clark SG, Gertler FB, Tessier-Lavigne M, Bargmann CI (2006) MIG-10/lamellipodin and AGE-1/PI3K promote axon guidance and outgrowth in response to slit and netrin. Curr Biol 16:854-862.

Colon-Ramos DA, Margeta MA, Shen K (2007) Glia promote local synaptogenesis through UNC-6 (netrin) signaling in C. elegans. Science 318:103-106

Dickson BJ (2002) Molecular mechanisms of axon guidance. Science 298:1959-1964.

Dodd RB, Drickamer K (2001) Lectin-like proteins in model organisms: implications for evolution of carbohydrate-binding activity. Glycobiology 11:71R-79R.

Drickamer K (1999) C-type lectin-like domains. Curr Opin Struct Biol 9:585-590.

Drickamer K, Dodd RB (1999) C-type lectin-like domains in Caenorhabditis elegans: predictions from the complete genome sequence. Glycobiology 9:1357-1369.

Fraser AG, Kamath RS, Zipperlen P, Martinez-Campos M, Sohrmann M, Ahringer J (2000) Functional genomic analysis of C. elegans chromosome I by systematic RNA interference. Nature 408:325-330.

Fujisawa K, Wrana JL, Culotti JG (2007) The slit receptor EVA-1 coactivates a SAX-3/Robo mediated guidance signal in C. elegans. Science 317:1934-1938.

Gitai Z, Yu TW, Lundquist EA, Tessier-Lavigne M, Bargmann CI (2003) The netrin receptor UNC-40/DCC stimulates axon attraction and outgrowth through enabled and, in parallel, Rac and UNC-115/AbLIM. Neuron 37:53-65.

Grill B, Bienvenut WV, Brown HM, Ackley BD, Quadroni M, Jin Y (2007) C. elegans RPM-1 regulates axon termination and synaptogenesis through the Rab GEF GLO-4 and the Rab GTPase GLO-1. Neuron 55:587-601.

Hallam SJ, Jin Y (1998) lin-14 regulates the timing of synaptic remodelling in Caenorhabditis elegans. Nature 395:78-82.

Hao JC, Yu TW, Fujisawa K, Culotti JG, Gengyo-Ando K, Mitani S, Moulder G, Barstead R, Tessier-Lavigne M, Bargmann CI (2001) C. elegans slit acts in midline, dorsal-ventral, and anterior-posterior guidance via the SAX-3/Robo receptor. Neuron 32:25-38.

Hedgecock EM, Culotti JG, Hall DH (1990) The unc-5, unc-6, and unc-40 genes guide circumferential migrations of pioneer axons and mesodermal cells on the epidermis in C. elegans. Neuron 4:61-85.

Hong K, Hinck L, Nishiyama M, Poo MM, Tessier-Lavigne M, Stein E (1999) A ligand-gated association between cytoplasmic domains of UNC5 and DCC family receptors converts netrin-induced growth cone attraction to repulsion. Cell 97:927-941.

Hutter H (2003) Extracellular cues and pioneers act together to guide axons in the ventral cord of C. elegans. Development 130:5307-5318.

Leung-Hagesteijn C, Spence AM, Stern BD, Zhou Y, Su MW, Hedgecock EM, Culotti JG (1992) UNC-5, a transmembrane protein with immunoglob- ulin and thrombospondin type 1 domains, guides cell and pioneer axon migrations in C. elegans. Cell 71:289-299.

Levy-Strumpf N, Culotti JG (2007) VAB-8, UNC-73 and MIG-2 regulate axon polarity and cell migration functions of UNC-40 in C. elegans. Nat Neurosci 10:161-168.

Li H, Kulkarni G, Wadsworth W (2008) RPM-1, a Caenorhabditis elegans protein that functions in presynaptic differentiation, negatively regulates axon outgrowth by controlling SAX-3/robo and UNC-5/UNC5 activity. J Neurosci 28:3595-3603.

Ma L, Tessier-Lavigne M (2007) Dual branch-promoting and branchrepelling actions of Slit/Robo signaling on peripheral and central branches of developing sensory axons. J Neurosci 27:6843-6851.

Mello C, Fire A (1995) DNA transformation. Methods Cell Biol 48:451-482.

Nakata K, Abrams B, Grill B, Goncharov A, Huang X, Chisholm AD, Jin Y (2005) Regulation of a DLK-1 and p38 MAP kinase pathway by the ubiquitin ligase RPM-1 is required for presynaptic development. Cell 120:407-420.

Nonet ML (1999) Visualization of synaptic specializations in live C. elegans with synaptic vesicle protein-GFP fusions. J Neurosci Methods 89:33-40.

Quinn CC, Pfeil DS, Chen E, Stovall EL, Harden MV, Gavin MK, Forrester WC, Ryder EF, Soto MC, Wadsworth WG (2006) UNC-6/netrin and SLT-1/slit guidance cues orient axon outgrowth mediated by MIG-10/ RIAM/lamellipodin. Curr Biol 16:845-853.

Ren XC, Kim S, Fox E, Hedgecock EM, Wadsworth WG (1999) Role of netrin UNC-6 in patterning the longitudinal nerves of Caenorhabditis elegans. J Neurobiol 39:107-118.

Schaefer AM, Hadwiger GD, Nonet ML (2000) rpm-1, a conserved neuronal gene that regulates targeting and synaptogenesis in C. elegans. Neuron 26:345-356.

Stein E, Tessier-Lavigne M (2001) Hierarchical organization of guidance receptors: silencing of netrin attraction by slit through a Robo/DCC receptor complex. Science 291:1928-1938.

Tessier-Lavigne M, Goodman CS (1996) The molecular biology of axon guidance. Science 274:1123-1133.

Wadsworth WG, Hedgecock EM (1996) Hierarchical guidance cues in the developing nervous system of C. elegans. BioEssays 18:355-362.

Wadsworth WG, Bhatt H, Hedgecock EM (1996) Neuroglia and pioneer neurons express UNC-6 to provide global and local netrin cues for guiding migrations in C. elegans. Neuron 16:35-46.

Wang KH, Brose K, Arnott D, Kidd T, Goodman CS, Henzel W, TessierLavigne M (1999) Biochemical purification of a mammalian slit protein as a positive regulator of sensory axon elongation and branching. Cell 96:771-784.

Watari-Goshima N, Ogura K, Wolf FW, Goshima Y, Garriga G (2007) C. elegans VAB-8 and UNC-73 regulate the SAX-3 receptor to direct cell and growth-cone migrations. Nat Neurosci 10:169-176.

Yu TW, Hao JC, Lim W, Tessier-Lavigne M, Bargmann CI (2002) Shared receptors in axon guidance: SAX-3/Robo signals via UNC-34/Enabled and a Netrin-independent UNC-40/DCC function. Nat Neurosci 5:1147-1154.

Zelensky AN, Gready JE (2003) Comparative analysis of structural properties of the C-type-lectin-like domain (CTLD). Proteins 52:466-477.

Zelensky AN, Gready JE (2005) The C-type lectin-like domain superfamily. FEBS J 272:6179-6217.

Zhen M, Huang X, Bamber B, Jin Y (2000) Regulation of presynaptic terminal organization by C. elegans RPM-1, a putative guanine nucleotide exchanger with a RING-H2 finger domain. Neuron 26:331-343. 